\title{
DESIGN, ANALYSIS, AND FABRICATION OF THE APT CAVITIES*
}

\author{
S. Atencio, B. Campbell, K. Chan, A. Cimabue, W. Clark, G. Ellis, R. Gentzlinger ${ }^{\#}$, H. Haagenstad, \\ D. Hammon, W. B Haynes, J. P. Kelley, F. Krawczyk, M. Manzo, F. Martinez, H. Martinez, \\ J. McClellen, J. Mitchell, R. Mitchell, D.I. Montoya, D.R. Montoya, J. Moss, S. Quintana, \\ A. Rendon, B. Rusnak, D. Schrage, F. Smith, M. Trujillo, LANL, Los Alamos, NM \\ J. Kuzminski, General Atomics, Los Alamos, NM
}

\begin{abstract}
The design for the superconducting cavities of the highenergy accelerator for the Accelerator Production of Tritium (APT) project has been extensively changed in the last two years. The original accelerator design incorporated 0.48 and 0.71 beta cavities with stiffeners, and a stainless-steel helium vessel. Today, the accelerator uses unstiffened 0.64 and 0.82 beta cavities, and a titanium helium vessel. This paper discusses the design process, including the supporting analyses, prototype cavities built, and the decisions made to support the current design. The design of the integrated cavity-helium vessel is presented. Future plans and testing for design validation are also discussed.
\end{abstract}

\section{INTRODUCTION}

The original APT machine proposed in the fall of 1995 included a room-temperature accelerator. A team was created to investigate the viability of using a superconducting accelerator for the high-energy end. A candidate design was developed [1] using two different ( $\beta=0.48$ and 0.71 ) cavities for beam energies between 100 $\mathrm{MeV}$ to $1200 \mathrm{MeV}$. The cavities were designed with a three-degree and five-degree wall slopes, respectively, which maximized the radio frequency (RF) performance while still allowing the acid to drain during chemical polishing. A stainless-steel liquid-helium containment vessel surrounding the cavity was incorporated into the design. Since there was little performance and manufacturing experience with low beta elliptical superconducting RF structures, a program to build four prototype single-cell cavities was undertaken. The cavities were manufactured at Los Alamos. The multi-cell cavities would be manufactured by US and European companies. The cavities would then be tested in the RFstructures laboratory at Los Alamos to see if a low-beta cavity could meet the program performance requirements.

During fabrication of the cavities, the accelerator architecture was revised to include three different beta cavities. The beta of the $\beta=0.71$ cavity was reduced to $\beta=0.64$ and a beta of 0.82 was added. The energy range of the superconducting high-energy accelerator was now
$100 \mathrm{MeV}$ to $1700 \mathrm{MeV}$. The fabrication of the $\beta=0.71$ cavity was stopped and the tooling was shelved. A $\beta=0.64$ single-cell cavity was designed and fabricated.

The test results of the cavities were encouraging [2], but the fabrication costs were high. Modifications to the design, for enhancing the mechanical strength/stiffness while trading off RF performance, were undertaken. A compromise was reached, where the predicted $\mathrm{RF}$ performance [3] of the $\beta=0.64$ cavity was slightly reduced ( $\sim 4 \%$ in field level due to increased $\mathrm{B}_{\text {peak }}$ ); the $\beta=0.82$ cavity performance under these criteria was affected by $<1 \%$, while the mechanical design became much simpler and easier to manufacture. The $\beta=0.48$ cavity was dropped from the accelerator architecture and the $\beta=0.64$ and $\beta=0.82$ cavities were redesigned. The energy range of the superconducting high-energy accelerator was now $240 \mathrm{MeV}$ to $1700 \mathrm{MeV}$. The helium vessel material was changed from stainless steel to titanium. With a slight reduction in the predicted cavity performance for the new $\beta=0.64$ design (with the new wall slope), and with the program schedule having the delivery of the 5-cell cavities on the critical path, a singlecell cavity-test program was not undertaken.

\section{CAVITY DESIGN \& FABRICATION}

\subsection{Original Cavity Design}

The original goal for the high-energy superconducting accelerator was to use off-the-shelf technology. No research and development was to be utilized in creating the accelerator design. Some engineering development would be required because, unlike an electron accelerator of $\beta=1.00, \beta<1.00$ cavities were needed. The APT cavity manufacturing plan was to use pure niobium sheet (RRR 250) and either press or spin it into the elliptical shape commonly used in electron accelerators. Other methods, such as niobium sputtering on copper, were considered too risky and therefore were not investigated. To maintain a reasonable accelerator length, two power couplers per cavity approach was adopted.

The three-degree to seven-degree wall slope resulting from the original design maximized performance and still

\footnotetext{
"Work supported by DOE under contract No. DE-AC04-96AL89607.

\#Email: gentzlinger@lanl.gov
} 
allowed the chemicals to drain from the cavity after polishing.

The five-degree slope of the $\beta=0.64$ cavity wall did not provide adequate stiffness or strength [4]. The applied pressures during the cool-down of the cavity would cause excessive yielding in the cavity. A $\beta=1.00$ cavity has more curvature and a smaller conical section than a $\beta=0.64$ cavity, which is more efficient at supporting the applied pressures. The conical section of the $\beta=0.64$ cavity is almost a flat plate when a five-degree slope is used.

The walls of the 0.48 and 0.64 beta cavities would yield significantly without any reinforcement. The original cavity design had cylindrical stiffeners between the cavity cells and a conical stiffener at each end of the cavity. The stiffeners are perforated with large diameter holes to allow the liquid helium to reach the cavity wall. The stiffeners allow the first mechanical resonant frequency of the cavity to be above the desired $100 \mathrm{~Hz}$. A stainless-steel helium vessel was installed over the niobium cavity. With the conical stiffeners covering up the beamtubes, the only place to fasten the vessel was the beamtube flanges. The five ports, (two RF power input couplers, two higher order mode couplers (HOM), and one RF pick-up) had to penetrate the helium vessel. Bellows were incorporated at the flange of each port to allow for the differential thermal contraction between the niobium cavity and the stainless-steel helium vessel.

The prototype stiffened, single-cell cavity was expensive and time consuming to fabricate. The APT machine would have been too expensive to fabricate if this cavity approach was adopted for the high-energy accelerator.

\subsection{Second Generation Cavity}

The second approach was to redesign the cavity with a revised cone angle to increase the stiffness. The more the cone angle could be increased (three to five to 10 degrees), the lower the resulting stresses would be. Increasing the angle too much would reduce the radius at the equator, raising the possibility of multipacting. A compromise of 10 degrees was adopted for both the 0.64 and 0.82 beta cavities. This slope allowed the stresses to be below the room temperature yield stress of 7,000 psi without the additional stiffeners. The 2.2 atm applied pressure from the cryogenic system during cool-down gave the smallest margin between the yield strength and the calculated state of stress in the cavity. The $2.2 \mathrm{~atm}$ is higher than what was originally used. The $3.0 \mathrm{~atm}$ at cryogenic temperature is not a problem with yield strength, since the yield increases by a factor of 10 at low temperature. Fracture toughness may be an issue at low temperatures and is being investigated. The cavity could still withstand the pressures without the additional stiffeners. However, the thickness had to be increased to 4.0 and $3.5 \mathrm{~mm}$ for the 0.64 and 0.82 beta cavities, respectively. The increased thickness added cost to the cavity fabrication, but it was less than adding the cost of the stiffeners.

With removal of the stiffeners, the five cavity ports could now be external to the helium vessel. To achieve this, the vessel would be attached to the beamtube. The original stainless-steel vessel design had a welded joint between the helium vessel and the port flanges. If the cavity ports were to be outside the helium vessel, a method of attaching the vessel to the niobium beamtube would have to be developed. Stainless steel could be attached to the niobium beamtubes by using a nioro ( $82 \%$ gold and $18 \%$ nickel) braze. The braze works quite well (the method is used for attaching the stainless-steel conflat $^{\mathrm{TM}}$ flanges to the niobium beamtubes), giving at least a 4000 psi shear stress capability.

Titanium is soluble in niobium and these metals can be electron-beam welded together. This approach ensures super fluid helium will not leak from the helium vessel. Titanium also has a coefficient of thermal expansion similar to that of niobium. This reduces the thermal stresses during cool down and reduces the requirement on the tuning range of the tuner. Titanium will not have any residual magnetic fields in the welds, therefore, it will not decrease the quality factor $(\mathrm{Q})$ of the cavity, thereby increasing the load on the cryogenic system.

The original requirement of having the lowest mechanical resonant frequency of $100 \mathrm{~Hz}$ could not be achieved with the current cavity design. It was decided that the microphonics issue would be resolved by evaluating the response of the cavity to the input power spectrum density of the APT plant or an equivalent. If the cavity's response was unacceptable, the cavity could be mechanically coupled to the helium vessel. This would be cheaper than adding stiffeners to the cavity. Coupling of the cavity to the helium vessel can be explained by reviewing the design of the helium vessel.

The original helium vessel had a tear-drop shape and was made from unalloyed grade 2 titanium. This allowed the vessel to encompass the cavity and have a minimal amount of liquid-helium storage. The bulkheads on each end of the vessel were machined from plates of titanium. The tuner end bulkhead had a flexure integrally machined into it. This gave compliance to the head, allowing the cavity to be tuned. The other end bulkhead had triangular stiffeners welded between it and the niobium beamtubes to reduce the stresses at the interface. The $2.2 \mathrm{~atm}$ design condition caused the almost flat section of the tear-drop shape to have unacceptable stresses. Stiffeners were needed in this region to lower the stresses to an acceptable level. Support rods were added internally to the vessel to strengthen and stiffen the two bulkheads to react the tuning loads. After the tuning range of the cavity tuner was increased to incorporate a $500 \mathrm{kHz}$ cool-down uncertainty (the $\beta=0.64$ cavity has a tuning sensitivity of $319 \mathrm{~Hz} /$ micron), the flexure design was scrapped. Then, after reviewing cleanliness issues and ideas of how best to 
couple the cavity to the vessel, the helium vessel was redesigned.

\subsection{Final Cavity Design}

The new and final helium vessel design (Figure 1) incorporates a vessel within a vessel concept and is made out of unalloyed grade 2 (yield strength of $40 \mathrm{Ksi}$ ) titanium. The inner vessel provides the strength and stiffness to support the cavity and its tuner. The outer vessel provides the storage volume for the liquid helium.

The tuner is supported off of one bulkhead of the inner vessel, having its loads transmitted down the vessel to the far bulkhead and then to its attached adjustable struts. The struts provide a load path for the tuning loads to return back to the cavity. This design provides a stiff path to react tuning loads against. The compliance of the second bulkhead would be too great if the struts were omitted, which causes the tuner to deflect the helium vessel instead of the cavity.

An edge-welded titanium bellows was added between the bulkhead and the niobium beamtubes on each end of the cavity. The bellows on one end allows the cavity beamtube to move relative to the helium vessel during the actuation of the tuner. The other bellows has two purposes. First, it minimizes the impact the manufacturing tolerances will have on the cavity's tuning range. The envelope for the bellows is quite limited due to the proximity of the cavity ports to the end half-cell, therefore, the stroke will be small. If the helium vessel and the cavity had significant variations from their desired design lengths, the range of the bellows would be significantly reduced. The second bellows allows the adjustable struts to relocate the vessel relative to the cavity. Second, the purpose of the second bellows is to decouple the cavity from the helium vessel. The cavity will be supported at one end by the power couplers. A statically indeterminate structure would arise if the cavity were welded directly to the independently supported helium vessel. This could be a significant problem during cool-down. The bellows will allow the cavity to move relative to the helium vessel. In the future, the second bellows may be eliminated if it is found unnecessary during cavity testing.

The inner vessel is perforated to allow the liquid helium to stay in contact with the cavity. The holes are located in-between the cavity cells. Complete rings are located at each of the five cells. Bumpers or spacers could be added at these locations to support the cavity if microphonics becomes an issue. The cavity will go through some significant testing to determine if bumpers or supports will be required. Note: if the bellows were located on the outside diameter of the inner vessel, the cavity could not be supported off the vessel.

The outer helium vessel provides the storage volume of the liquid helium. The vessel holds approximately 125 liters of liquid helium for the $\beta=0.64$ cavity. The vessel requires a titanium-to-stainless-steel transition [5] joint to go between the titanium vessel and the stainless-steel conflat $^{\mathrm{TM}}$ flanges. Preliminary trials of this joint were successful using inertial welding between the two materials.

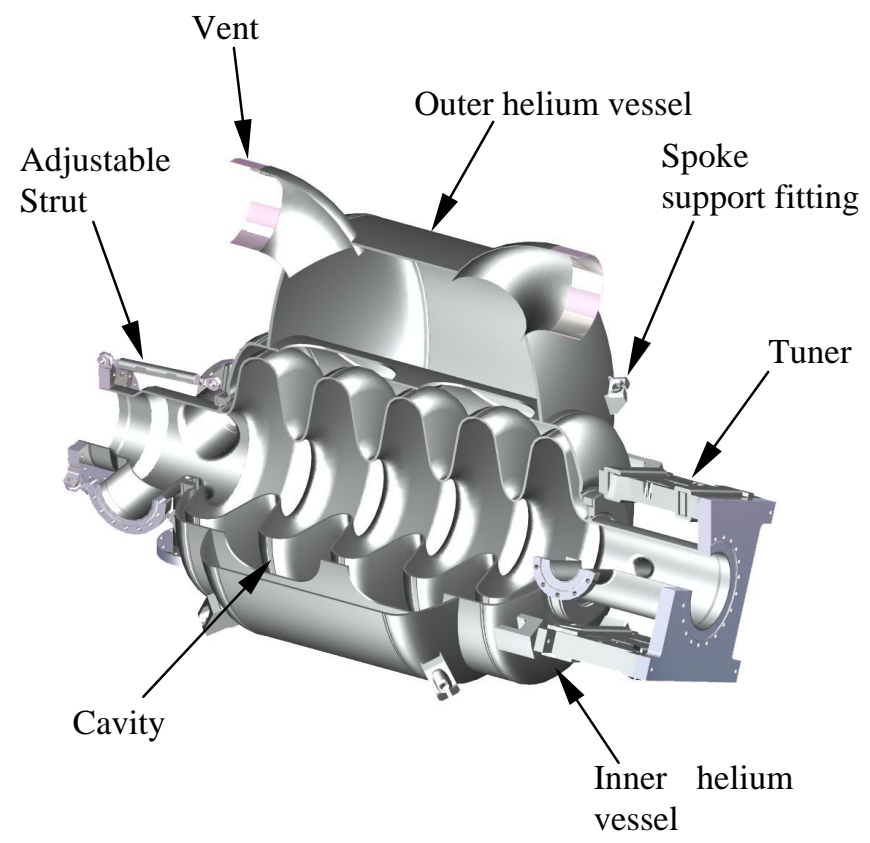

Figure 1: The current $\beta=0.64$ cavity and helium vessel

\section{ADDITIONAL WORK}

The designs of the cavity and helium vessel were presented to the Los Alamos Pressure Vessel Review Committee during the fall of 1998. The committee is responsible for reviewing the engineering and operations of cryogenic and pressure vessel systems. Their approval is required before any operations can begin. The designs of both components would be accepted as long as the design passed a fracture toughness analysis. This analysis could not begin without some fracture toughness data being generated. A contract was placed at the High Magnetic Lab at Florida State University to develop fracture toughness data for niobium and unalloyed grade 2 titanium. This data should be available by June of 1999 .

\section{REFERENCES}

[1] K.C. Dominic Chan et al., LANL Memo: LA-UR-954045

[2] W.B.Haynes et al., LANL Memo: LA-UR-98-682

[3] F. Krawczyk et al., LANL Memo: LA-UR-97-1700

[4] D. Schrage \& E. Swensen, LANL Memo: AOT-1:95180

[5] M. Cola et al., "Inertia Friction Welded Joints between a Titanium Vessel and a Stainless Steel Cryogenic Plumbing System for the APT", for PAC “99", New York City, (Mar 99) 